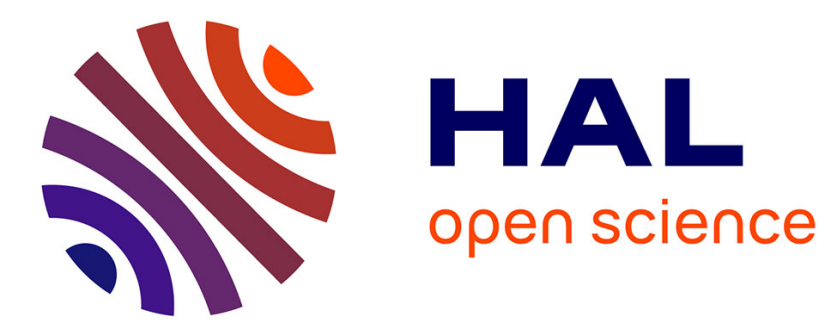

\title{
Mixed-norm estimates for paraproducts
}

Cristina Benea, Camil Muscalu

\section{- To cite this version:}

Cristina Benea, Camil Muscalu. Mixed-norm estimates for paraproducts. Analyse des Equations aux

Dérivées Partielles Roscoff, 30 Mai au 3 Juin 2016, May 2016, Roscoff, France. hal-01403035

\section{HAL Id: hal-01403035 \\ https://hal.science/hal-01403035}

Submitted on 13 Dec 2016

HAL is a multi-disciplinary open access archive for the deposit and dissemination of scientific research documents, whether they are published or not. The documents may come from teaching and research institutions in France or abroad, or from public or private research centers.
L'archive ouverte pluridisciplinaire HAL, est destinée au dépôt et à la diffusion de documents scientifiques de niveau recherche, publiés ou non, émanant des établissements d'enseignement et de recherche français ou étrangers, des laboratoires publics ou privés. 


\title{
MIXED-NORM ESTIMATES FOR PARAPRODUCTS
}

\author{
CRISTINA BENEA AND CAMIL MUSCALU
}

\begin{abstract}
We present a new approach to the study of singular multi-parameter multilinear Fourier multipliers via multiple vector-valued inequalities. This summarizes some of our results from 1 and 2 . The main example is the bi-parameter paraproduct $\Pi \otimes \Pi$, for which we prove estimates within the whole range of admissible Lebesgue estimates
\end{abstract}

\section{INTRODUCTION}

Our aim is to present a few recent tools from harmonic analysis that could prove useful in the study of nonlinear differential equations. These involve multilinear Littlewood-Paley theory in several variables on mixed norm $L^{p}$ spaces

$$
\|f\|_{L_{x}^{p} L_{t}^{q}}:=\|\| f\left\|_{L_{t}^{q}}\right\|_{L_{x}^{p}}:=\left(\int_{\mathbb{R}}\left(\int_{\mathbb{R}}|f(x, t)|^{q} d t\right)^{p / q} d x\right)^{1 / p} .
$$

Our main results will be formulated as Leibniz rules in mixed norm $L^{p}$ spaces, but other multipliers can be treated similarly. Of great importance to us are the less conventional cases involving quasi-Banach target spaces $\left(s_{1}, s_{2}\right.$ can be $<1$ in Theorem 1) or $L^{\infty}$ input spaces (we allow for $p_{j}=\infty$ or $q_{j}=\infty$ ). From the technical point of view, these are more interesting because they are not direct consequences of the linear Littlewood-Paley theory.

Theorem 1. For any $\alpha, \beta>0$

$$
\begin{aligned}
\left\|D_{1}^{\alpha} D_{2}^{\beta}(f \cdot g)\right\|_{L_{x}^{s_{1}} L_{y}^{s_{2}}} & \lesssim\left\|D_{1}^{\alpha} D_{2}^{\beta} f\right\|_{L_{x}^{p_{1}} L_{y}^{p_{2}}} \cdot\|g\|_{L_{x}^{q_{1}} L_{y}^{q_{2}}}+\|f\|_{L_{x}^{p_{3}} L_{y}^{p_{4}}} \cdot\left\|D_{1}^{\alpha} D_{2}^{\beta} g\right\|_{L_{x}^{q_{3} L_{y}^{q_{4}}}} \\
& +\left\|D_{1}^{\alpha} f\right\|_{L_{x}^{p_{5} L_{y}^{p_{6}}}} \cdot\left\|D_{2}^{\beta} g\right\|_{L_{x}^{q_{5} L_{y}^{q_{6}}}}+\left\|D_{2}^{\beta} f\right\|_{L_{x}^{p_{7}} L_{y}^{p_{8}}} \cdot\left\|D_{1}^{\alpha} g\right\|_{L_{x}^{q_{7} L_{y}^{q_{8}}}},
\end{aligned}
$$

whenever $1<p_{j}, q_{j} \leq \infty, \max \left(\frac{1}{2}, \frac{1}{1+\alpha}, \frac{1}{1+\beta}\right)<s_{1}, s_{2}<\infty$ and so that the indices satisfy the natural Hölder-type conditions.

A result of a similar type appeared in [9], as an important tool in establishing local wellposedness for the generalized Korteweg-de Vries equation. This is a dispersive, nonlinear equation given by

$$
\left\{\begin{array}{l}
\frac{\partial u}{\partial t}+\frac{\partial^{3} u}{\partial x^{3}}+u^{k} \frac{\partial u}{\partial x}=0, \quad t, x \in \mathbb{R}, k \in \mathbb{Z}^{+} \\
u(x, 0)=u_{0}(x)
\end{array}\right.
$$

In order to prove existence, the authors use the contraction principle, but to be able to do so, they need to construct a suitable Banach space. The norm of the Banach space involves mixed $L^{p}$ norms of fractional derivatives in the first variable $D_{1}^{\alpha}$, and the Leibniz rule employed in this paper is

$$
\left\|D_{1}^{\alpha}(f \cdot g)-f \cdot D_{1}^{\alpha} g-D_{1}^{\alpha} f \cdot g\right\|_{L_{x}^{p} L_{t}^{q}} \lesssim C\left\|D_{1}^{\alpha_{1}} f\right\|_{L_{x}^{p_{1}} L_{t}^{q_{1}}}\left\|D_{1}^{\alpha_{2}} g\right\|_{L_{x}^{p_{2} L_{t}^{q_{2}}}} .
$$


Here $\alpha \in(0,1), \alpha_{1}+\alpha_{2}=\alpha$ and $\frac{1}{p_{1}}+\frac{1}{p_{2}}=\frac{1}{p}, \frac{1}{q_{1}}+\frac{1}{q_{2}}=\frac{1}{q}$. Also, $p, p_{1}, p_{2}, q, q_{1}, q_{2} \in(1, \infty)$, but one can allow $q_{1}=\infty$ if $\alpha_{1}=0$.

The fractional derivatives appear as a consequence of the smoothness requirement on the initial data: $u_{0}$ is assumed to be in some Sobolev space $H^{\alpha}(\mathbb{R})$, where $\alpha$ depends on the value of $k$ in (1).

The paraproduct of two functions (termed para-multiplication operator in [3], where they were first used in the study of non-linear differential equations) is a bilinear operator associated to the Littlewood-Paley decompositions of two functions $f$ and $g$. For any $k \in \mathbb{Z}$, the Fourier projection of $f$ onto the shell $|\xi| \sim 2^{k}$ is denoted $Q_{k} f(x):=f * \psi_{k}(x)$, while the projection onto $|\xi| \leq 2^{k}$ is $P_{k} f(x):=f * \varphi_{k}(x)$. Then it is not difficult to see that

$$
f \cdot g=\sum_{k}\left(\left(f * \psi_{k}\right) \cdot\left(g * \psi_{k}\right)\right) * \varphi_{k}+\sum_{k}\left(\left(f * \varphi_{k}\right) \cdot\left(g * \psi_{k}\right)\right) * \psi_{k}+\sum_{k}\left(\left(f * \psi_{k}\right) \cdot\left(g * \varphi_{k}\right)\right) * \psi_{k} .
$$

Any of the three elements on the right hand side of the expressions above is called a classical paraproduct and is denoted $\Pi(f, g)$. They also play the role of mollifiers: instead of estimating $D^{\alpha}(f \cdot g)$, we estimate $D^{\alpha}(\Pi(f, g))$. When a derivative hits $\psi_{k}$ or $\varphi_{k}$, this becomes equivalent to multiplication by $2^{k \alpha}$; and correspondingly, $2^{k \alpha} \psi_{k}(x)=D^{\alpha} \tilde{\tilde{\psi}}_{k}(x)$. We will see in Section 2 how Theorem 1 can be deduced from results on bi-parameter paraproducts.

The concept of paraproduct extends readily to bilinear Fourier multipliers:

Theorem A (Coifman, Meyer [5]). Any bilinear multiplier operator associated to a symbol $m(\xi, \eta)$ satisfying $\left|\partial^{\alpha} m(\xi, \eta)\right| \lesssim|(\xi, \eta)|^{-\alpha}$ for sufficiently many multi-indices $\alpha$, maps $L^{p}(\mathbb{R}) \times L^{q}(\mathbb{R})$ into $L^{s}(\mathbb{R})$ provided that $1<p, q \leq \infty, \frac{1}{2}<s<\infty$, and $\frac{1}{p}+\frac{1}{q}=\frac{1}{s}$.

However, we will not be working with classical paraproducts as above, but with disretized paraproducts, which are associated to a collection $\mathcal{J}$ of dyadic intervals:

$$
\Pi_{\mathcal{J}}(f, g)(x)=\sum_{I \in \mathcal{J}} c_{I} \frac{1}{|I|^{\frac{1}{2}}}\left\langle f, \varphi_{I}\right\rangle\left\langle g, \psi_{I}\right\rangle \psi_{I}(x),
$$

where the functions $\psi_{I}$ and $\varphi_{I}$ are so that $\operatorname{supp} \hat{\psi}_{I} \subseteq\left[\frac{1}{\mid T}, \frac{2}{|T|}\right], \operatorname{supp} \hat{\varphi}_{I} \subseteq\left[0, \frac{1}{|I|}\right]$, are $L^{2}$-normalized and decay fast away from $I$.

The discretized paraproducts above can be used as a black box in the study of several other bilinear multipliers singular at a point, as we will see in Section 2. The information on the Fourier multiplier will be encoded in the decay of the double Fourier coefficients of its restriction to the Whitney squares associated to the point singularity.

The approach based on discretized operators, presented in various instances in [13], developed with the study of the bilinear Hilbert transform operator, defined by

$$
\begin{aligned}
B H T(f, g)(x): & =p \cdot v \cdot \int_{\mathbb{R}} f(x-t) g(x+t) \frac{d t}{t} \\
& =C \int_{\mathbb{R}^{2}} \hat{f}(\xi) \hat{g}(\eta) \operatorname{sgn}(\xi-\eta) e^{2 \pi i x(\xi+\eta)} d \xi d \eta . .
\end{aligned}
$$

This operator was introduced by Alberto Calderón, in relation to the analysis of the Cauchy integral on Lipschitz curves and to what are now called Calderón's commutators. 
Indeed, can be written as

$$
\begin{aligned}
C_{1} f(x) & =-p \cdot v \cdot \int_{\mathbb{R}} \frac{A(x+t)-A(x)}{t} f(x+t) \frac{d t}{t}= \\
& =-p \cdot v \cdot \int_{\mathbb{R}}\left(\int_{0}^{1} a(x+\alpha t) d \alpha\right) f(x+t) \frac{d t}{t},
\end{aligned}
$$

where $a(x)=A^{\prime}(x) \in L^{\infty}$.

A question that remained open for nearly thirty years, long after the Cauchy integral on Lipschitz curves was comprehended, was whether BHT: $L^{p} \times L^{\infty} \rightarrow L^{p}$, for $1<p<\infty$. This question was eventually answered in the late 90 's, reviving the field of time-frequency analysis (which priorly consisted of Carleson's celebrated proof of the pointwise convergence of Fourier series [4], and Fefferman's reinterpretation of the latter 7]).

Theorem B (Lacey, Thiele [10]). BHT is a bounded bilinear operator from $L^{p} \times L^{q}$ into $L^{s}$, for any $1<p, q \leq \infty, 0<s<\infty$, satisfying $\frac{1}{p}+\frac{1}{q}=\frac{1}{s}$ and $\frac{2}{3}<s<\infty$.

In some sense, proving quasi-Banach valued results for multilinear operators is not as much about understanding the behavior of $T(f, g)$ in a space where the triangle inequality doesn't hold, as it is about understanding the admissible input spaces for the operator. This is especially relevant for the bilinear Hilbert transform operator above; it is still not clear if the operator is bounded for values of $s$ between $\frac{1}{2}$ and $\frac{2}{3}$. This would correspond to both $p$ and $q$ being strictly less that 2 , loosing some of the orthogonality (in frequency) properties that are so significant in the proof.

Later on, the result was extended to multilinear multipliers singular along a subspace of dimension $k$ :

Theorem C (Muscalu, Tao, Thiele [14]). Let $\Gamma \subset \mathbb{R}^{n}$ be a non-degenerate subspace of dimension $k$, with $0 \leq k<\frac{n+1}{2}$, and let $T_{m}$ be an $n$-linear operator given by

$$
T_{m}\left(f_{1}, \ldots, f_{n}\right)(x)=\int_{\mathbb{R}^{n}} m\left(\xi_{1}, \ldots, \xi_{n}\right) \hat{f}_{1}\left(\xi_{1}\right) \cdot \ldots \cdot \hat{f}_{n}\left(\xi_{n}\right) e^{2 \pi i x\left(\xi_{1}+\ldots+\xi_{n}\right)} d \xi_{1} \ldots d \xi_{n} .
$$

Above, the multiplier $m$ satisfies the estimates

$$
\left|\partial_{\xi}^{\alpha} m(\xi)\right| \lesssim \operatorname{dist}(\xi, \Gamma)^{-|\alpha|}
$$

for all partial derivatives up to some finite order. Then $T: L^{p_{1}} \times \ldots \times L^{p_{n}} \rightarrow L^{p}$ whenever $\frac{1}{p_{1}}+\ldots+\frac{1}{p_{n}}=\frac{1}{p}, 1<p_{j} \leq \infty, \frac{1}{n}<p<\infty$ and moreover

$$
\frac{1}{p_{i_{1}}}+\ldots+\frac{1}{p_{r}}<\frac{n+1-2 k+r}{2}
$$

for all $1 \leq i_{1}<\ldots<i_{r} \leq n$ and $1 \leq r \leq n$ (here we set $p_{n+1}=p$ ).

The non-degeneracy condition requires $\Gamma$ to be a graph over any $k$ variables; otherwise, the problem reduces to a lower dimensional one, and as a consequence, the range of boundedness will be different.

The case $k=0$ corresponds to the Coifman-Meyer theorem $\mathrm{A}$, while $k=1, n=2$ to the Lacey-Thiele theorem B,

There exist also certain results for multipliers that depend also on the spacial variable:

Theorem D (Bernicot, [3]). Consider a smooth symbol $a\left(x, \xi_{1}, \xi_{2}\right)$ satisfying

$$
\left|\partial_{x}^{\beta} \partial_{\xi}^{\alpha} a(x, \xi)\right| \lesssim(1+\operatorname{dist}(\xi, \Gamma))^{-|\alpha|},
$$


for sufficiently many indices $\alpha$ and $\beta$. Then the bilinear operator associated to this symbol satisfies the same estimates as the bilinear Hilbert transform of Theorem $B$.

In order to prove Theorem 1, we need to estimate bi-parameter paraproducts:

$$
\Pi \otimes \Pi(f, g)(x, y)=\sum_{k, l}\left[\left(f * \varphi_{k} \otimes \varphi_{l}\right) \cdot\left(g * \psi_{k} \otimes \psi_{l}\right)\right] *\left(\psi_{k} \otimes \psi_{l}\right)(x, y),
$$

and the remaining similar eight terms obtained by permuting the $\psi_{k}, \varphi_{k}, \psi_{l}, \varphi_{l}$ functions.

The first result along these lines was proved by Journé in [8], who in fact developed a theory for linear bi-parameter Calderón-Zygmund operators. However, Journé did prove that $\Pi \otimes \Pi: L^{2}\left(\mathbb{R}^{2}\right) \times L^{\infty}\left(\mathbb{R}^{2}\right) \rightarrow L^{2}\left(\mathbb{R}^{2}\right)$. The general result was proved later in [11]:

Theorem E (Muscalu, Pipher, Tao, Thiele [11]). $\Pi \otimes \Pi$ is a bounded operator from $L^{p}\left(\mathbb{R}^{2}\right) \times L^{q}\left(\mathbb{R}^{2}\right)$ into $L^{s}\left(\mathbb{R}^{2}\right)$ provided that $1<p, q \leq \infty, \frac{1}{p}+\frac{1}{q}=\frac{1}{s}$, and $0<s<\infty$.

In [1] together with [2], we managed to prove the following result:

Theorem 2. Let $1<p_{i}, q_{i} \leq \infty$ and $\frac{1}{2}<s_{i}<\infty$, be so that $\frac{1}{p_{i}}+\frac{1}{q_{i}}=\frac{1}{s_{i}}$ for any index $i=1,2$. Then the bi-parameter paraproduct $\Pi \otimes \Pi$ satisfies the following mixed norm estimates:

$$
\Pi \otimes \Pi: L_{x}^{p_{1}} L_{y}^{p_{2}} \times L_{x}^{q_{1}} L_{y}^{q_{2}} \rightarrow L_{x}^{s_{1}} L_{y}^{s_{2}} .
$$

A similar result holds for multi-parameter paraproducts $\Pi \otimes \ldots \otimes \Pi$, if the lebesgue exponents are strictly between 1 and $\infty$ (although we can allow the outer-most exponent to be between $\frac{1}{2}$ and 1 as well).

Theorem E was proved using discretized paraproducts in both variables, that is $\Pi_{\mathcal{J}} \otimes \Pi_{\mathcal{\jmath}}$. This is not the approach we take; the mixed norm $L^{p}$ spaces already have a vector-valued structure buit-in. Instead, we use some multiple vector-valued estimates for paraproducts (which we deduce from similar estimates for the discretized paraproducts). In Section 3 we point out to the bridge between vector-valued and multi-parameter theory.

First, in [1], we treated the case $1 \leq s_{2}<\infty$, corresponding to a Banach case, by developing a way of proving multiple vector-valued estimates for certain bilinear operators ( paraproducts and $B H T$ ). In [2], we extended these methods to quasi-Banach spaces, allowing for values of $s_{2}$ strictly between $\frac{1}{2}$ and $\infty$.

We should mention that a similar result, within a smaller Banach range, was proved independently around the same time in [6].

The vector-valued result, on which our proof relies, is the following theorem:

Theorem 3. Consider the tuples $R_{1}=\left(r_{1}^{1}, \ldots, r_{1}^{n}\right), R_{2}=\left(r_{2}^{1}, \ldots, r_{2}^{n}\right)$ and $R=\left(r^{1}, \ldots, r^{n}\right)$ satisfying for every $1 \leq j \leq n: 1<r_{1}^{j}, r_{2}^{j} \leq \infty, \frac{1}{2}<r^{j}<\infty$, and $\frac{1}{r_{1}^{j}}+\frac{1}{r_{2}^{j}}=\frac{1}{r^{j}}$. Then the paraproduct $\Pi$ satisfies the estimates

$$
\Pi: L^{p}\left(\mathbb{R} ; L^{R_{1}}(\mathcal{W}, \mu)\right) \times L^{q}\left(\mathbb{R} ; L^{R_{2}}(\mathcal{W}, \mu)\right) \rightarrow L^{s}\left(\mathbb{R} ; L^{R}(\mathcal{W}, \mu)\right),
$$

for any $1<p, q \leq \infty, \frac{1}{2}<s<\infty$ with $\frac{1}{p}+\frac{1}{q}=\frac{1}{s}$.

The $L^{R}$ norm on $(\mathcal{W}, \Sigma, \mu)=\left(\prod_{j=1}^{n} \mathcal{W}_{j}, \prod_{j=1}^{n} \Sigma_{j}, \prod_{j=1}^{n} \mu_{j}\right)$ is defined as

$$
\|\vec{f}\|_{L^{R}(\mathcal{W}, \mu)}:=\left(\int_{\mathcal{W}_{1}} \ldots\left(\int_{\mathcal{W}_{n}}\left|\vec{f}\left(w_{1}, \ldots, w_{n}\right)\right|^{r^{n}} d \mu_{n}\left(w_{n}\right)\right)^{r^{n-1} / r^{n}} \ldots d \mu_{1}\left(w_{1}\right)\right)^{1 / r^{1}}
$$




\section{Reduction to Discretized paraproducts}

We briefly explain how to reduce the study of bilinear Fourier multipliers as in Theorem A. to that of discretized paraproducts. But first we illustrate this reduction for classical paraproducts. The trilinear form associated to the paraproducts can be written as

$$
\begin{aligned}
\Lambda_{\Pi}(f, g, h) & =\sum_{k} \int_{\mathbb{R}}\left(f * \varphi_{k}\right)(x)\left(g * \psi_{k}\right)(x)\left(h * \psi_{k}\right)(x) d x \\
& =\sum_{k \in \mathbb{Z}} 2^{-k} \sum_{n \in \mathbb{Z}} \int_{0}^{1}\left(f * \varphi_{k}\right)\left(2^{-k}(n+\beta)\right)\left(g * \psi_{k}\right)\left(^{-k}(n+\beta)\right)\left(h * \psi_{k}\right)\left(^{-k}(n+\beta)\right) d \beta \\
& =\sum_{k} \int_{0}^{1} \sum_{|I|=2^{-k}} \frac{1}{|I|^{1 / 2}}\left\langle f, \varphi_{I}^{\beta}\right\rangle\left\langle g, \psi_{I}^{\beta}\right\rangle\left\langle h, \psi_{I}^{\beta}\right\rangle \\
& =\int_{0}^{1} \sum_{I} \frac{1}{|I|^{1 / 2}}\left\langle f, \varphi_{I}^{\beta}\right\rangle\left\langle g, \psi_{I}^{\beta}\right\rangle\left\langle h, \psi_{I}^{\beta}\right\rangle .
\end{aligned}
$$

The functions $\psi_{I}^{\beta}$ are given by $\psi_{I}^{\beta}(t)=2^{-k / 2} \overline{\psi_{k}\left(2^{-k}(n+\beta)-t\right)}$, and similarly for $\varphi_{I}^{\beta}$. They are $L^{2}$ normalized and verify the properties in the definition of the discretized paraproducts. The parameter beta varies within the compact interval $[0,1]$, and for that reason its presence is of no consequence.

Regarding the paraproduct $\Pi(f, g)=\sum_{k} P_{k}\left(Q_{k} f \cdot Q_{k} g\right)$, for every $k \in \mathbb{Z}, P_{k}\left(Q_{k} f \cdot Q_{k} g\right)$ is supported in frequency inside the set $|\xi| \sim 2^{k},|\eta| \sim 2^{k}$, which can be written as a union of a finite number of Whitney squares with respect to the origin. That is, squares in the frequency plane $\xi \eta$, so that the diameter of the box is comparable to the distance to the singularity.

Conversely, let $m(\xi$, eta $)$ be a symbol satisfying $\left|\partial^{\alpha} m(\xi, \eta)\right| \lesssim|(\xi, \eta)|^{-\alpha}$ for sufficiently many multi-indices $\alpha$. We can write $m$ as

$$
m(\xi, \eta)=\sum_{Q} m(\xi, \eta) \cdot \phi_{Q}(\xi, \eta)=\sum_{Q} m_{Q}(\xi, \eta)
$$

where $Q$ runs over Whitney squares associated to the point singularity, and $\phi_{Q}$ represent a smooth partition of unity associated to the collection of suqares $Q$. Every $m_{Q}$ is smooth and supported mainly on $Q$, hence we can consider its Fourier series:

$$
m_{Q}(\xi, \eta)=\sum_{n_{1}, n_{2}} c_{n_{1}, n_{2}}^{Q} e^{\frac{2 \pi i n_{1} \xi}{|Q|}} e^{\frac{2 \pi i n_{2} \xi}{|Q|}}=\sum_{n_{1}, n_{2}} c_{n_{1}, n_{2}}^{Q} e^{\frac{2 \pi i n_{1} \xi}{|Q|}} e^{\frac{2 \pi i n_{2} \xi}{|Q|}} \phi_{Q_{1}}(\xi) \phi_{Q_{2}}(\eta),
$$

where this time $\phi_{Q_{1}}(\xi)$ is a smooth function supported on $\frac{11}{10} Q_{1}$, which is constantly equal to 1 on $Q_{1}$. (Here we write $Q=Q_{1} \times Q_{2}$ ). The smoothness of the symbol $m$ is reflected in the decay of the Fourier coefficients, which is uniform in $Q$ :

$$
\left|c_{n_{1}, n_{2}}^{Q}\right| \lesssim\left(1+\left|n_{1}\right|+\left|n_{2}\right|\right)^{-M}
$$

In fact, $c_{n_{1}, n_{2}}^{Q}$ only depends on the diameter of the square, hence we write $c_{n_{1}, n_{2}}^{k}$ for a square of diameter comparable to $2^{k}$.

Then we have

$$
\begin{aligned}
T_{m}(f, g)(x) & =\sum_{n_{1}, n_{2}} \sum_{k} c_{n_{1}, n_{2}}^{k}\left(\left(f * \varphi_{k, n_{1}}\right) \cdot\left(g * \psi_{k, n_{2}}\right)\right) * \psi_{k}(x) \\
& + \text { like terms. }
\end{aligned}
$$


The functions $\psi_{k, n_{1}}$ are the modulated functions $\psi_{k}$, which correspond to a shift in the spatial variable. Then expressions of the form

$$
\sum_{k} c_{n_{1}, n_{2}}^{k}\left(\left(f * \varphi_{k, n_{1}}\right) \cdot\left(g * \psi_{k, n_{2}}\right)\right) * \psi_{k}(x)
$$

represent shifted paraproducts, which can be reduced again to discretized paraproducts. The shifts with respect to the parameters $n_{1}$ and $n_{2}$ produce a dependency of these variables, but which is acceptable in the end thanks to the fast decay of the coefficients $c_{n_{1}, n_{2}}^{k}$.

The Leibniz rule from Theorem 1. Using paraproducts, $D_{1}^{\alpha} D_{2}^{\beta}(f, g)(x, y)$ can be represented as a sum of paraproducts applied to $D_{1}^{\alpha} f$ and $D_{2}^{\beta} g$ and to similar terms (this explains the numerous terms in Theorem 10.

When the derivatives are applied to the term $\sum_{k, l} P_{k} P_{l}\left(Q_{k} Q_{l} f \cdot Q_{k} Q_{l} g\right)$, we obtain

$$
D_{1}^{\alpha} D_{2}^{\beta}(\Pi(f, g))(x, y)=\sum_{k, l}\left[\left(f * \psi_{k} \otimes \psi_{l}\right) \cdot\left(g * \psi_{k} \otimes \psi_{l}\right)\right] *\left(2^{k \alpha} \tilde{\varphi}_{k} \otimes 2^{l \beta} \tilde{\varphi}_{l}\right)(x, y) .
$$

The functions $\tilde{\varphi}_{k}$ and $\tilde{\varphi}_{l}$ have a slower decay than $\varphi_{k}, \varphi_{l}$ :

$$
\hat{\tilde{\varphi}}_{k}(\xi)=\frac{|\xi|^{\alpha}}{2^{k \alpha}} \hat{\varphi}_{k}, \quad \hat{\tilde{\varphi}}_{l}(\eta)=\frac{|\eta|^{\beta}}{2^{\beta}} \hat{\varphi}_{l} .
$$

The simple lack of smoothness at the origin causes $\tilde{\varphi}$ to decay as $(1+|x|)^{-1-\alpha}$ (here we have $\tilde{\varphi}$ so that $\left.\tilde{\varphi}_{k}(x)=2^{k} \tilde{\varphi}\left(2^{k} x\right)\right)$.

The $\tilde{\varphi}_{k}$ and $\tilde{\varphi}_{l}$ will be again decomposed in Fourier series in frequency as before, and we are reduce to the study of the operator

$$
\sum_{n_{1}, n_{2}} \sum_{k} c_{n_{1}, n_{2}}^{k, l}\left(\left(f * \psi_{k, n_{1}} \otimes \psi_{l, n_{2}}\right) \cdot\left(g * \psi_{k, n_{1}} \otimes \psi_{l, n_{2}}\right)\right) * \varphi_{k} \otimes \varphi_{l}(x, y)
$$

where this time the coefficients satisfy uniformly in $k$ and $l$ the estimate

$$
\left|c_{n_{1}, n_{2}}^{k, l}\right| \lesssim \frac{1}{\left(1+\left|n_{1}\right|\right)^{(1+\alpha)}} \cdot \frac{1}{\left(1+\left|n_{2}\right|\right)^{(1+\beta)}} .
$$

The expression in (6) is essentially

$$
\sum_{n_{1}, n_{2}} c_{n_{1}, n_{2}} \tilde{\Pi} \otimes \tilde{\Pi}(f, g)(x, y)
$$

and we will need to estimate this in $L_{x}^{s_{1}} L_{y}^{s_{2}}$. Using the subadditivity of $\|\cdot\|_{L_{x}^{s_{1}} L_{y}^{s_{2}}}^{s_{0}}$, where $s_{0}=\min \left(1, s_{1}, s_{2}\right)$, and assuming the estimate in Theorem 2 , Theorem 1 is reduced to the summability of

$$
\sum_{n_{1}, n_{2}} \frac{1}{\left(1+\left|n_{1}\right|\right)^{s_{0}(1+\alpha)}} \cdot \frac{1}{\left(1+\left|n_{2}\right|\right)^{s_{0}(1+\beta)}} .
$$

The latter is true provided $s_{0}(1+\alpha)>1$ and $s_{0}(1+\beta)>1$, i.e. if

$$
\min \left(1, s_{1}, s_{2}\right)>\max \frac{1}{1+\alpha}, \frac{1}{1+\beta} .
$$




\section{From multiple VeCtor-VAlued operators to MUlti-PARAmeter operators}

The study of multi-parameter operators in harmonic analysis is not an easy task. The reason for that, at least partly, is the more complicated characterization of the $B M O$ space in higher dimensions, which plays a very important role in the study of bilinear operators as well. This can avoided however, by regarding multi-parameter operators as (some multiple) vector-valued operators, an approach we are going to take.

Correspondingly, vector-valued extensions of an operator $T$ can be regarded as mixednorm estimates for the bi-parameter operator $T \otimes I d$ (in the second component, nothing happens). This is a useful point of view because it also suggests that bi-parameter operators can be understood in a way similar to the treatment of vector-valued extensions.

Sometimes in time-frequency analysis, multi-parameter operators behave unexpectedly. In [11, it was noticed that even though $\Pi \otimes \Pi$ is bounded on all admissible $L^{p}$ spaces, $B H T \otimes B H T=\infty$ a.e. The range of boundedness for $\Pi \otimes B H T$ was only recently understood in [16] and [1].

The paraproducts on the other hand have a special "splitting" property. Recall that $Q_{k}$ is the Littlewood-Paley projection onto $\left\{|\xi| \sim 2^{k}\right\}$ (which is really the convolution with $\left.\psi_{k}(\cdot)\right)$, and $P_{k}$ is the projection onto $\left\{|\xi| \leq 2^{k}\right\}$, corresponding to the convolution with $\varphi_{k}$. Then we can regard paraproducts as being expressions of the form

$$
\sum_{k} Q_{k}\left(P_{k} f \cdot Q_{k} g\right)(x, y) \quad, \sum_{k} Q_{k}\left(Q_{k} f \cdot P_{k} g\right)(x, y) \quad \text { or } \sum_{k} P_{k}\left(Q_{k} f \cdot Q_{k} g\right)(x, y) .
$$

It is important for us that the outer-most function $\hat{\varphi}_{k}\left(\xi_{1}+\xi_{2}\right)$ and $\hat{\psi}_{k}\left(\xi_{1}+\xi_{2}\right)$ are identically equal to 1 on the supports of $\hat{\psi}_{k}\left(\xi_{1}\right) \cdot \hat{\psi}_{k}\left(\xi_{2}\right)$ and $\hat{\psi}_{k}\left(\xi_{1}\right) \cdot \hat{\varphi}_{k}\left(\xi_{2}\right)$ respectively. This can always be achieved with the price of an extra decomposition.

Proposition 1. Let $T_{m}: L^{p}\left(\mathbb{R}^{n}\right) \times L^{q}\left(\mathbb{R}^{n}\right) \rightarrow L^{r}\left(\mathbb{R}^{n}\right)$ be a bilinear operator with smooth symbol $m$, and $\Pi: L^{p}(\mathbb{R}) \times L^{q}(\mathbb{R}) \rightarrow L^{r}(\mathbb{R})$ a paraproduct as described above.

(1) If $\Pi$ is given by $\sum_{k} Q_{k}\left(P_{k} f \cdot Q_{k} g\right)(x, y)$, then

$$
\left(T_{m} \otimes \Pi\right)(f, g)(x, y)=\sum_{k} Q_{k}^{2}\left(T_{m}\left(P_{k}^{y} f, Q_{k}^{y} g\right)\right)(x)=\sum_{k} T_{m}\left(P_{k}^{y} f, Q_{k}^{y} g\right)(x) .
$$

(2) If $\Pi$ is given by $\sum_{k} P_{k}\left(Q_{k} f \cdot Q_{k} g\right)(x, y)$, then

$$
\left(T_{m} \otimes \Pi\right)(f, g)(x, y)=\sum_{k} P_{k}^{2}\left(T_{m}\left(Q_{k}^{y} f, Q_{k}^{y} g\right)\right)(x)=\sum_{k} T_{m}\left(Q_{k}^{y} f, Q_{k}^{y} g\right)(x) .
$$

Here we need to explain the notation: $Q_{k}^{2}$ denotes the projection onto $\left|\xi_{2}\right| \sim 2^{k}$ in the second variable, and $P_{k}^{y} f$ is a function of $x$ only, with the variable $y$ fixed. The exact formulas are

$$
\begin{aligned}
& P_{k}^{y} f(x)=\int_{\mathbb{R}} \varphi_{k}(s) f(x, y-s) d s, \quad P_{k}^{2} f(x, y)=\int_{\mathbb{R}} \varphi_{k}(s) f(x, y-s) d s, \\
& Q_{k}^{y} f(x)=\int_{\mathbb{R}} \psi_{k}(s) f(x, y-s) d s, \quad Q_{k}^{2} f(x, y)=\int_{\mathbb{R}} \psi_{k}(s) f(x, y-s) d s .
\end{aligned}
$$

With this observation at hand, we can present our approach to multiparameter paraproducts. 
Sketch of proof for Theorem 2. Now we show how to deduce the results of Theorem 2, for any $\frac{1}{2}<s_{1}, s_{2}<\infty$.

If $\Pi_{y}$, the paraproduct acting on the variable $y$ is of the form

$$
\Pi_{y}(\cdot, \cdot)=\sum_{k} Q_{k}\left(P_{k}(\cdot), Q_{k}(\cdot)\right),
$$

then we can write $\Pi \otimes \Pi$ as $\Pi \otimes \Pi(f, g)(x, y)=\sum_{k} Q_{k}^{2} \Pi\left(P_{k}^{y}, Q_{k}^{y}\right)(x)$.

A less known result, which plays an important role in our approach, is the following lemma, which appears in [15]:

Lemma 1. Let $f \in \mathcal{S}\left(\mathbb{R}^{n}\right)$, and $1 \leq l \leq n$, and $\left\{i_{1}, \ldots i_{l}\right\} \subset\{1, \ldots, n\}$. Then

$$
\|f\|_{L^{p}} \lesssim\left\|\left(\sum_{k_{1}, \ldots, k_{l}}\left|Q_{k_{1}}^{i_{1}} \ldots Q_{k_{l}}^{i_{l}} f\right|^{2}\right)^{1 / 2}\right\|_{L^{p}}
$$

for any $0<p<\infty$.

This is saying that the $L^{p}$ norm of $f$ is bounded by the $L^{p}$ norm of the square function, for any $0<p<\infty$. The reverse statement is used more often, but it only holds for $1<p<\infty$. Also, a Banach-valued result holds, and it will be needed for the special case $\frac{1}{2}<s_{1}<1$, and $p_{2}=\infty$ or $q_{2}=\infty$, when the Banach space concerned is $L^{1}$.

We have

$$
\begin{aligned}
& \|\| \sum_{k} Q_{k}^{2} \Pi\left(P_{k}^{y}, Q_{k}^{y}\right)(x)\left\|_{L_{y}^{s_{2}}}\right\|_{L_{x}^{s_{1}}} \lesssim\|\|\left(\sum_{k}\left|\Pi\left(P_{k}^{y}, Q_{k}^{y}\right)(x)\right|^{2}\right)^{1 / 2}\left\|_{L_{y}^{s_{2}}}\right\|_{L_{x}^{s_{1}}} \\
& \lesssim\|\| \sup _{k}\left|P_{k}^{y} f(x)\right|\left\|_{L_{y}^{p_{2}}}\right\|_{L_{x}^{p_{1}}} \cdot\|\|\left(\sum_{k}\left|Q_{k}^{y} g(x)\right|^{2}\right)^{1 / 2}\left\|_{L_{y}^{q_{2}}}\right\|_{L_{x}^{q_{1}}} .
\end{aligned}
$$

In the above inequality we used the multiple vector-valued estimate

$$
\Pi_{x}: L_{x}^{p_{1}}\left(L_{y}^{p_{2}}\left(\ell^{\infty}\right)\right) \times L_{x}^{q_{1}}\left(L_{y}^{q_{2}}\left(\ell^{2}\right)\right) \rightarrow L_{x}^{s_{1}}\left(L_{y}^{s_{2}}\left(\ell^{2}\right)\right),
$$

which is a consequence of Theorem 3 .

On the last line of (9), we recognize a maximal operator in big \|\| $\sup _{k}\left|P_{k}^{y} f(x)\right|\left\|_{L_{y}^{p_{2}}}\right\|_{L_{x}^{p_{1}}}$ and a square function in \|\|$\left(\sum_{k}\left|Q_{k}^{y} g(x)\right|^{2}\right)^{1 / 2}\left\|_{L_{y}^{q_{2}}}\right\|_{L_{x}^{q_{1}}}$. The latter is not bounded on $L^{\infty}$, leaving out the case $q_{2}=\infty$.

If $1 \leq s_{1}, s_{2}<\infty$, the case when $p_{2}=\infty$ or $q_{2}=\infty$ can be obtained by duality: the dual of $L_{x}^{p} L_{y}^{q}$ is $L_{x}^{p^{\prime}} L_{y}^{q^{\prime}}$. More more difficult however is the case when $\frac{1}{2}<s_{1}<1$ and $p_{2}=\infty$ or $q_{2}=\infty$, and needs to be dealt with differently. Indeed, we treat it similarly to vector-valued extensions for operators: we prove sharp estimates for the localized version

$$
\Pi_{I_{0}}^{F, G, H^{\prime}} \otimes \Pi: L_{x}^{\infty} L_{y}^{\infty} \times L_{x}^{q} L_{y}^{q} \rightarrow L_{x}^{q} L_{y}^{q}
$$

with sharp operatorial bounds that depend on the dyadic interval $I_{0}$, and the fixed sets $F, G, H^{\prime}$. Then the mixed norm $L^{p}$ estimates are deduced through careful stopping times.

On the other hand, if $\Pi_{y}$ is of the form

$$
\Pi_{y}(\cdot, \cdot)=\sum_{k} P_{k}\left(Q_{k}(\cdot), Q_{k}(\cdot)\right),
$$

we can write $\Pi \otimes \Pi$ as $\Pi \otimes \Pi(f, g)(x, y)=\sum_{k} P_{k}^{2} \Pi\left(Q_{k}^{y}, Q_{k}^{y}\right)(x)$. Then the boundedness of $\Pi \otimes \Pi$ in $L_{x}^{s_{1}} L_{y}^{s_{2}}$ follows from the vector-valued inequality

$$
\Pi_{x}: L_{x}^{p_{1}}\left(L_{y}^{p_{2}}\left(\ell^{2}\right)\right) \times L_{x}^{q_{1}}\left(L_{y}^{q_{2}}\left(\ell^{2}\right)\right) \rightarrow L_{x}^{s_{1}}\left(L_{y}^{s_{2}}\left(\ell^{2}\right)\right) .
$$




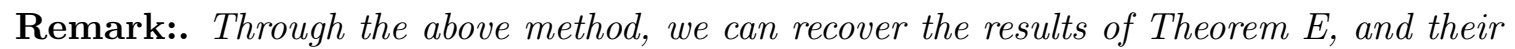
multi-parameter equivalents from [12].

\section{A FEW COMMENTS ON QUASI-BANACH SPACES}

Even though for $0<p<1$, the triangle inequality is missing in $L^{p}$ spaces, the boundedness of a (multi-) linear operator on such spaces can still be proved thanks to Marcinkiewicz interpolation theorem, which is true also for multi-sublinear operators. In this situation, the dual of $L^{p}$ is $\{0\}$ for non-atomic spaces(such as $L^{p}\left(\mathbb{R}^{n}\right)$ ), or a complicated space containing $\ell^{\infty}$ in the case of an atomic space such as $\ell^{p}$. We will not attempt to dualize the $L^{p}$ quasinorm, but instead the $L^{p, i n f t y}$ quasinorm, which could seem surprising.

Using generalized restricted type dualization, we have

$$
\|f\|_{p, \infty} \sim \sup _{E, 0<|E|<\infty} \inf _{\substack{E^{\prime} \subseteq E \\ \text { major subset }}} \frac{\left|\left\langle f, \mathbf{1}_{E^{\prime}}\right\rangle\right|}{|E|^{1-\frac{1}{p}}},
$$

where we say $E^{\prime}$ is a major subset of $E$ if $E^{\prime} \subseteq E$ and $\left|E^{\prime}\right| \geq|E| / 2$.

More generally, we have

$$
\|f\|_{p, \infty} \sim \sup _{0<|E|<\infty} \inf _{\substack{\tilde{E} \subseteq E \\ \text { major subset }}} \frac{\left\|f \cdot \mathbf{1}_{\tilde{E}}\right\|_{r}}{|E|^{\frac{1}{r}-\frac{1}{p}} .}
$$

This means that, given a set $E$ of finite measure, we can in fact remove an exceptional subset whose measure is relatively small (which can represent the set where the maximal function of $f$ is too large, for example), and estimate $f$ on the complement of the exceptional set. Such a technique is extremely useful, but there might be certain technicalities involved and one needs to exercise care. If we want to evaluate $\|T f\|_{p_{0}, \infty}$ and $\|T f\|_{p_{1}, \infty}$, and from this deduce estimates for $\|T\|_{L^{p} \rightarrow L^{p}}$ via a Marcinkiewicz interpolation theorem, the exceptional set should be independent of the function $f$.

The type of interpolation most suitable to this techniques is the generalized restrictedtype interpolation (which is an adaptation of Marcinkiewicz's ideas). The principle behind it is that whatever is true for characteristic functions should also hold for function in $L^{p}$ spaces. We present a bilinear theorem which is often used in time-frequency analysis:

Proposition 2. Let $(p, q, s)$ be a tuple so that $\frac{1}{2}<s<\infty, 1<p, q<\infty$ and $\frac{1}{s}=\frac{1}{p}+\frac{1}{q}$, and $T$ a bilinear operator satisfying the restricted type estimate:

for any sets of finite measure $E_{1}$ and $E_{2}$, and any functions $f, g$ so that $|f(x)| \leq \mathbf{1}_{E_{1}}(x)$ and $|g(x)| \leq \mathbf{1}_{E_{2}}(x)$ respectively, the estimate

$$
\|T(f, g)\|_{\tilde{s}, \infty} \leq K_{s_{1}, s_{2}, \tilde{s}}\left|E_{1}\right|^{1 / s_{1}}\left|E_{2}\right|^{1 / s_{2}}
$$

holds for all tuples $\left(s_{1}, s_{2}, \tilde{s}\right)$ satisfying $\frac{1}{s_{1}}+\frac{1}{s_{2}}=\frac{1}{\tilde{s}}$ in a neighborhood of $(p, q, s)$, with the constant $K_{s_{1}, s_{2}, \tilde{s}}$ depending continuously on $s_{1}, s_{2}, \tilde{s}$.

Then $T$ is of strong type $(p, q, s)$, in the sense that

$$
\|T(f, g)\|_{s} \leq K_{p, q, s}\|f\|_{p}\|g\|_{q}
$$

for any sequences of functions for which the RHS is finite.

The interpolation theorem above extends easily to the framework of multiple Banach or quasi-Banach spaces; the conditions $|f(x)| \leq \mathbf{1}_{E_{1}},|g(x)| \leq \mathbf{1}_{E_{2}}(x)$ are replaced by 
$\|\vec{f}(x)\|_{L^{R_{1}(\mathcal{W}, \mu)}} \leq \mathbf{1}_{E_{1}}(x)$ and $\|\vec{g}(x)\|_{L^{R_{2}(\mathcal{W}, \mu)}} \leq \mathbf{1}_{E_{2}}(x)$ respectively. Moreover, the measure spaces can be arbitrary, for example if we want to prove:

$$
\|\| T(\vec{f}, \vec{g})\left\|_{L^{R}(\mathcal{W}, \mu)}\right\|_{L^{s}(\nu)} \leq K_{p, q, s}\|\| \vec{f}\left\|_{L^{R_{1}}}\right\|_{L^{p}\left(\nu_{1}\right)}\|\| \vec{g}\left\|_{L^{R_{2}}}\right\|_{L^{q}\left(\nu_{2}\right)} .
$$

Remark:. In [2], identity (11) plays an essential role. In proving Theorem 3, it was important for us to linearize the operator $\left(\sum_{k}\left|T\left(f_{k}, g_{k}\right)\right|^{r}\right)^{1 / r}$ for values of $r<1$. This could not be achieved pointwise (the dual of $\ell^{r}$ in this case is unknown), but using (11) we were able to linearize the $L^{p, \infty}$ norm of $\left(\sum_{k}\left|T\left(f_{k}, g_{k}\right)\right|^{r}\right)^{1 / r}$.

\section{REFERENCES}

[1] C. Benea And C. Muscalu, Multiple vector valued inequalities via the helicoidal method. http://arxiv.org/pdf/1511.04948v1.pdf, 2015.

[2] Quasi-banach valued inequalities via the helicoidal method. https://arxiv.org/pdf/1609.01090v1.pdf, 2016.

[3] F. BeRniCOT, Local estimates and global continuities in Lebesgue spaces for bilinear operators, Anal. PDE, 1 (2008), pp. 1-27.

[4] L. CARLeson, On convergence and growth of partial sums of Fourier series, Acta. Math., (1966), pp. $135-157$.

[5] R. Coifman And Y. Meyer, Wavelets, Calderón-Zygmund Operators and Multilinear Operators, Cambridge University Press, 1997.

[6] F. DI Plinio AND Y. OU, Banach-valued multilinear singular integrals. http://arxiv.org/abs/1506.05827, 2015. Online; accessed June 2016.

[7] C. Fefferman, Pointwise convergence of Fourier series, The Annals of Mathematics, (1973), pp. 551571.

[8] J.-L. Journé, Calderón-Zygmund operators on product spaces, Revista Matemtica Iberoamericana, 1 (1985), pp. 55-91.

[9] C. Kenig, G. Ponce, And L. Vega, Well-posedness and scattering results for the generalized Korteweg-de Vries equation via the contraction principle, Comm. Pure Appl. Math., (1993), pp. 527620.

[10] M. Lacey And C. Thiele, On Calderón's conjecture, Ann. of Math. (2), 149 (1999), pp. 475-496.

[11] C. Muscalu, J. Pipher, T. Tao, and C. Thiele, Bi-parameter paraproducts, Acta Mathematica, (2004), pp. 269-296.

[12] - Multi-parameter paraproducts, Rev. Mat. Iberoamericana, (2006), pp. 963-976.

[13] C. Muscalu And W. Schlag, Classical and Multilinear Harmonic Analysis, Cambridge University Press, 2013.

[14] C. Muscalu, T. TaO, And C. Thiele, Multi-linear operators given by singular multipliers, J. Amer. Math. Soc., (2002), pp. 469-496.

[15] Z. RuAn, Multi-parameter Hardy spaces via discrete Littlewood-Paley theory, Anal. Theory Appl., 26 (2010), pp. 122-139.

[16] P. Silva, Vector valued inequalities for families of bilinear Hilbert transforms and applications to biparameter problems, J. Lond. Math. Soc., (2014), pp. 695-724.

Cristina Benea, CNRS - Université de Nantes, Laboratoire Jean Leray, Nantes 44322, FRANCE

E-mail address: cristina.benea@univ-nantes.fr

Camil Muscalu, Department of Mathematics, Cornell University, Ithaca, Ny 14853, USA

E-mail address: camil@math.cornell.edu 